\title{
Extériorisation des potentialités génétiques du zébu Peulh sénégalais (Gobra)
}

\author{
par J.P. DENIS $\left({ }^{*}\right)$ et J. VALENZA $\left({ }^{*}\right)$
}

\begin{abstract}
RESUME
Trois taurillons appartenant à un lot d'animaux dont les potentialités génétiques ont été extériorisées par une alimentation rationnelle dès leur naissance sont abattus à 30,29 et 27 mois. Leurs poids respectifs sont de 589,556 et $440 \mathrm{~kg}$ représentant un gain moyen journalier de 632,620 et $510 \mathrm{~g}$. Les rendements sont de $64,7,62,8$ et 63,7 p. 100. Les carcasses obtenues sont supérieures à celles d'animaux provenant de l'élevage traditionnel ou de parc d'embouche.
\end{abstract}

La sélection du zébu Peulh sénégalais ou Gobra (**) — en vue de déterminer et d'exalter ses remarquables qualités bouchères, se poursuit au Centre de Recherches zootechniques de Dara-Djoloff, depuis 1954.

Effectuée en maintenant le troupeau d'étude dans des conditions de vie et d'entretien aussi proches que possible de celles qui caractérisent l'élevage extensif dans la région sahélienne du Ferlo, elle a déjà permis d'isoler quelques lignées dont les performances sont nettement supérieures à la moyenne, moyenne qui est, en outre, en constante augmentation.

Les potentialités zootechniques latentes du Gobra, tant du point de vue de la précocité que du rendement en viande, qui n'ont rien à envier à nombre de races perfectionnées, ont été effectivement mises en évidence en 1962 par REDON qui a montré que le jeune Gobra convenablement alimenté était susceptible de déve-

(*) Institut d'Elevage et de Médecine vétérinaire des Pays tropicaux, Centre de Recherches zootechniques de Dara-Djoloff; Laboratorre national de l'Elevage et de Recherches vétérinaires, Dakar-Hann.

(**) Le lecteur désireux de se documenter avec précision sur cette race pourra consulter utilement Ies articles déjà publiés dans la Revue, dont les références sont données dans la bibliographie de cet article. lopper des performances étonnantes par rapport aux très sévères conditions écologiques qui règnent dans l'aire de dispersion de cette variété de zébu Peulh.

C'est tant pour préciser les véritables possibilités d'extériorisation des potentialités de cette race que pour juger l'efficacité des travaux de sélection effectués à Dara qu'une étude complète dans ce sens a été entreprise en portant surtout l'action sur l'alimentation, qui, dans les conditions naturelles, constitue le facteur limitant essentiel et sur lequel il est possible d'agir le plus aisément.

Un certain nombre d'animaux ont donc reçu, dès leur naissance, en plus du lait maternel une alimentation équilibrée et adaptée à leurs besoins, mise à volonté à leur disposition jusqu'à ce qu'ils soient abattus, à des âges différents, entre 2 et 5 ans pour étude des carcasses et du rendement.

Cette étude ayant pour seul objet de préciser le potentiel créateur de viande du Gobra, les notions économiques, de prix de revient notamment, ont été délibérément laissées de côté. Le cas échéant, elles seront reprises pour fixer s'il est économiquement rentable de produire des bcufs de boucherie de haute qualité, dans le cadre du marché de la viande au Sénégal. 
Première partie

\section{CROISSANCE DE LA NAISSANCE AU SEVRAGE (6 MOIS)}

\section{MATERIEL - METHODES}

\section{Matériel}

Quinze veaux et 29 velles nés entre le 22 avril et le 24 septembre 1968 constituent le lot expérimental. Ces animaux ont été intégrés dans le lot d'extériorisation au fur et à mesure de leur naissance, c'est-à-dire sans tenir compte des caractéristiques des parents, des mères en particulier (vaches à différents degrés de sélection, primipares ou multipares).

\section{Alimentation}

De la naissance au sevrage les jeunes animaux reçoivent en complément du lait maternel, un concentré alimentaire titrant environ 0,90 UF et $125 / 130 \mathrm{~g}$ de matières azotées digestibles au $\mathrm{kg}$.

A partir du sevrage et jusqu'à deux ans. ces animaux sont mis en parc où ils ont à leur disposition, en plus du pâturage, le même concentré à volonté et dans la mesure du possible du foin récolté sur la station.

Ce concentré, présenté en granulés, a la composition suivante :

- maïs . . . . . . . . . $10 \quad \mathrm{~kg}$

— son de blé . . . . . . $38 \quad \mathrm{~kg}$

— son de maïs . . . . . . . $16 \quad \mathrm{~kg}$

— son de sorgho . . . . . $28 \quad \mathrm{~kg}$

- tourteau d'arachide . . . . $5,250 \mathrm{~kg}$

- carbonate de calcium . . . $2 \mathrm{~kg}$

- chlorure de sodium . . . . . $0,5 \quad \mathrm{~kg}$

— complément vitaminé . . . $0,250 \mathrm{~kg}$

Ce qui donne:

p. 1.000

- matière sèche

$905,8 \mathrm{~g}$

p. 1.000

de la mat. sèche

— matières minérales $53,9 \mathrm{~g}$

- matières organiques $946,1 \mathrm{~g}$

- matières grasses $64,6 \mathrm{~g}$

— matières azotées $(\mathrm{N} \times 6,25)$. 157,0 g

- matières cellulosiques (Wende) .

- extractif non azoté

$76,3 \mathrm{~g}$ $648,2 \mathrm{~g}$
- calcium $11,9 \mathrm{~g}$

- phosphore

$7,2 \mathrm{~g}$

Les mères pendant toute leur lactation reçoivent un $\mathrm{kg}$ par jour de ce même concentré.

A partir de deux ans, ces animaux recevront, en plus de la ration de base (foin et paille naturels de la station) et à volonté, un concentré titrant environ $0,8 \mathrm{UF}$ et $90 \mathrm{~g}$ de matières azotées digestibles au $\mathrm{kg}$.

\section{Observations effectuées}

Le poids et les mensurations classiques (périmètre thoracique, hauteur au garrot, longueur scapulo-ischiale, longueur et largeur de la tête, longueur de la croupe, largeur de la hanche, hauteur aux sangles) sont pris selon le rythme suivant :

- toutes les semaines jusqu'à 13 semaines;

— tous les 15 jours jusqu'à 6 mois;

- tous les mois jusqu’à 2 ans;

- tous les 3 mois jusqu'à 6 ans.

Le rendement en viande et la qualité des carcasses sont jugés régulièrement :

à 2 ans, 3 ans, 4 ans et 5 ans, trois mâles sont abattus (un parmi chaque classe de poids : faible, moyen, fort) et leur carcasse étudiée.

\section{Lot témoin}

Etant donné les différences significatives de poids aux mêmes âges en fonction du trimestre de naissance (1), il est impossible de prendre pour "lot témoin" les animaux nés en 1968 avant le 22 avril ou après le 24 septembre, qui représentent 66 p. 100 des naissances. Aussi ce lot est-il représenté par l'ensemble des animaux nés en 1965-1966 et 1967 qui recevaient, de leur naissance au sevrage, $500 \mathrm{~g}$ par jour d'un concentré de composition un peu différente, titrant environ 0,90 UF et $100 / 110 \mathrm{~g}$ de matières azotées digestibles au $\mathrm{kg}$.

\section{RESULTATS - DISCUSSION}

Seules les croissances pondérables moyennes de la naissance à 6 mois sont rapportées et figurent aux tableaux 1 et 2 .

\section{Mâles}

Les poids à la naissance et à un mois sont significativement différents entre les deux lots. 
TABLEAU $\mathrm{N}^{\circ} \mathrm{I}$

\begin{tabular}{|l|c|c|c|c|c|c|c|c|}
\hline \multirow{5}{*}{ Extếriorisation } & & Naissance & 1 mois & 2 mois & 3 mois & 4 mois & 5 mois & 6 mois \\
\cline { 2 - 9 } & $\mathrm{y}$ & 14 & 15 & 15 & 14 & 15 & 15 & 15 \\
& $\overline{\mathrm{x}}$ & 21,3 & 33,0 & 44,6 & 64,1 & 82,3 & 104 & 126,3 \\
& \pm & 1,6 & 3,2 & 4,3 & 7,5 & 9,3 & 11,6 & 12,2 \\
\hline \multirow{3}{*}{ Témoin } & $\mathrm{N}$ & 227 & 222 & 223 & 209 & 191 & 187 & 162 \\
& $\overline{\mathrm{x}}$ & 25,0 & 38,5 & 48,7 & 61,6 & 72,7 & 85,3 & 96,2 \\
\hline F calculé & \pm & 0,5 & 0,9 & 1,1 & 1,6 & 3,8 & 2,4 & 2,7 \\
\hline
\end{tabular}

F $0,05=3,89$

$F 0,01=6,76$

Cette supériorité du lot témoin est due, en grande partie, à la prise en compte des animaux nés aux $1^{\text {er }}$ et $4^{\circ}$ trimestres dont les poids à la naissance sont significativement supérieurs à ceux nés au cours des $2^{*}$ et $3^{e}$ trimestres (1), qui seuls constituent le lot expérimental.

A deux et trois mois la différence n'est plus significative. Le lat "extériorisé " a rattrapé son retard. De plus les animaux nés aux $2^{\mathrm{e}}$ et surtout $3^{e}$ trimestres ont un poids à 10 semaines supérieur aux autres.
A partir de 3 mois et jusqu'à 6 mois, la différence redevient significative mais à l'avantage du lot expérimental dont la croissance est supérieure au témoin.

Les croissances moyennes journalières sont respectivement de 475 et $406 \mathrm{~g}$ de 0 à 3 mois, 690 et $385 \mathrm{~g}$ de 3 à 6 mois, 585 et $395 \mathrm{~g}$ de la naissance au sevrage.

\section{Femelles}

De la naissance à deux mois la différence

TABLEAU $N^{\circ} \mathrm{II}$

\begin{tabular}{|c|c|c|c|c|c|c|c|c|}
\hline & & Naissance & 1 mois & 2 mois & 3 mois & 4 mois & 5 mois & 6 mois \\
\hline Extëriorisation & $\begin{array}{l}\mathrm{N} \\
\overline{\mathrm{x}} \\
\pm\end{array}$ & $\begin{array}{l}27 \\
22,7 \\
2,1\end{array}$ & $\begin{array}{l}29 \\
35,6 \\
3,1\end{array}$ & $\begin{array}{l}28 \\
47,7 \\
4,7\end{array}$ & $\begin{array}{l}2 B \\
63,6 \\
6,5\end{array}$ & $\begin{array}{l}28 \\
81,9 \\
7,4\end{array}$ & $\begin{array}{r}28 \\
101,0 \\
8,3\end{array}$ & $\begin{array}{r}28 \\
118,9 \\
8,5\end{array}$ \\
\hline Témoin & $\begin{array}{l}\bar{N} \\
\bar{x} \\
\pm\end{array}$ & $\begin{array}{r}233 \\
23,5 \\
0,6\end{array}$ & $\begin{array}{r}246 \\
35,9 \\
0,7\end{array}$ & $\begin{array}{r}242 \\
45,4 \\
1,0\end{array}$ & $\begin{array}{r}231 \\
58,1 \\
1,4\end{array}$ & $\begin{array}{r}215 \\
68,3 \\
1,6\end{array}$ & $\begin{array}{r}206 \\
78,5 \\
1,9\end{array}$ & $\begin{array}{r}200 \\
88,1 \\
2,2\end{array}$ \\
\hline F calculé & & 1,71 & 38,24 & 1,77 & 5,85 & 26,21 & 56,41 & 83,14 \\
\hline
\end{tabular}

F $0,05=3,89 \quad$ F $0,01=6,76$

entre les deux lots n'est pas significative. A partir du troisième mois, elle le devient et sa valeur est très forte au moment du sevrage.

Les croissances moyennes journalières sont respectivement de 416 et $365 \mathrm{~g}$ de 0 à 2 mois, 593 et $356 \mathrm{~g}$ de 2 à 6 mois, 535 et $342 \mathrm{~g}$ de la naissance au sevrage.

Certains animaux ont des croissances remarquables. C'est ainsi qu'on peut citer le cas d'un veau pesant $20 \mathrm{~kg}$ à la naissance, $77 \mathrm{~kg}$ à 3 mois et $167 \mathrm{~kg}$ à 6 mois (croît moyen journalier de $816 \mathrm{~g}$ de 0 à 6 mois et de $1 \mathrm{~kg}$ de 3 à
6 mois), d'une velle pesant $16 \mathrm{~kg}$ à la naissance, $50 \mathrm{~kg}$ à 2 mois et $148 \mathrm{~kg}$ à 6 mois (crô̂t moyen journalier de $733 \mathrm{~g}$ et de $816 \mathrm{~g}$ de 2 à 6 mois).

\section{CONCLUSION}

La distribution à volonté de concentré alimentaire de croissance à de jeunes zébus gobras dès leur naissance ne paraît pas indispensable pendant les deux ou trois premiers mois. Par contre, à partir du moment où la production 
laitière des vaches diminue et devient insuffisante, cette supplémentation à volonté est bénéfique et permet de sevrer à 6 mois des animaux d'un poids nettement supérieur à la moyenne ( $30 \mathrm{~kg}$ chez les mâles et les femelles).

Cette supplémentation fait ressortir une hétérogénéité entre les animaux quant à leur croissance et leur précocité, tant chez les mâles que chez les femelles. Par voie de conséquence, également, cette expérience confirme qu'une sélection efficace et rapide sur le plan de la précocité bouchère doit s'appuyer sur une supplémentation indispensable à partir du moment où la production laitière maternelle n'est plus suffisante pour assurer la croissance optimale du jeune. Pour le zébu gobra et dans les conditions de l'élevage sénégalais, cette supplémentation doit intervenir approximativement vers l'âge de 2 à 3 mois.

\section{Deuxième partie}

\section{CROISSANCE DE 6 A 24 MOIS}

Du sevrage à 24 mois le même concentré distribué à volonté a été fourni aux animaux. Le poids et les mensurations classiques ont été effectués tous les mois durant la période considérée.

\section{Lot témoin}

Les mêmes témoins que dans la première partie permettent de comparer les valeurs obtenues dans le lot extériorisé. Les calculs de signification entre le lot extériorisé et le lot témoin n'ont pas été effectués à partir de 6 mois, car les différences entre les performances des 2 lots sont trop différentes. Cependant pour donner une idée de cet écart, les poids aux âges caractéristiques sont donnés au tableau $\pi^{\circ} 3$ pour illustrer la croissance du lot témoin.

TABLEAU $\mathrm{N}^{\mathrm{a}}$ III

\begin{tabular}{|c|c|c|c|c|c|c|c|}
\hline & & 6 miois & 12 mois & 18 mois & 24 mois & 30 mois & 36 mois \\
\hline \multirow{3}{*}{ Mâles } & N & 162 & 199 & 145 & 95 & 68 & 65 \\
\hline & $\bar{x}$ & 96,2 & 144,0 & 196,60 & 259,60 & 312,6 & 364,3 \\
\hline & \pm & 2,7 & 4,0 & 6,1 & 7,8 & 9,6 & 13,3 \\
\hline \multirow{3}{*}{ Femelles } & $\mathrm{N}$ & 200 & 220 & 165 & 11 & 92 & 89 \\
\hline & $\bar{x}$ & 88,1 & 129,0 & 174,0 & 220,4 & 270,5 & 309,5 \\
\hline & \pm & 2,2 & 3,5 & 5,5 & 7,4 & 9,0 & 9,2 \\
\hline
\end{tabular}

\section{Résultats dans le lot extériorisé}

Seules les performances pondérales sont ici analysées et figurent au tableau $n^{0} 4$. En ce qui concerne les gains de poids successifs, ils figurent au tableau $\mathrm{n}^{\circ} 5$.

\section{1. Mâles}

La courbe de croissance des mâles Gobra jusqu'à 24 mois est pratiquement rectiligne, avec cependant un fléchissement à partir du $21^{\mathrm{e}}$ mois.

En effet du $6^{\mathrm{e}}$ au $21^{\mathrm{e}}$ mois, le gain de poids moyen journalier s'élève à $736 \mathrm{~g}$ et du $21^{\mathrm{e}}$ au $24^{\mathrm{e}}$ mois il n'est plus que de $360 \mathrm{~g}$. Pour l'ensemble de la période le gain journalier est de $673 \mathrm{~g}$.

\section{2. Femelles}

En ce qui concerne l'étude de l'évolution pondérale des femelles, il faut tenir compte du fait qu'elles ont été placées à 1a reproduction dès le mois de janvier 1970 et que 14 d'entre elles vêlent entre octobre et décembre (veaux d'un poids moyen important : $25 \mathrm{~kg}$ avec des extrêmes de 21 à $31 \mathrm{~kg}$ ).

Le gain de poids moyen journalier est de :

- $546 \mathrm{~g} \mathrm{du} 6^{\mathrm{e}}$ au $21^{\mathrm{e}}$ mois;

- $260 \mathrm{~g}$ du $21^{\mathrm{e}}$ au $24^{\mathrm{e}}$ mois;

- $500 \mathrm{~g} \mathrm{du} 6^{\mathrm{e}}$ au $24^{\mathrm{e}}$ mois. 
TABLEAU $\mathrm{N}^{\circ} \mathrm{IV}$

Croissance pondêrale des animaux extêriorisếs

\begin{tabular}{|c|c|c|c|c|c|c|c|c|c|c|c|c|c|c|c|c|c|c|c|c|}
\hline \multirow{4}{*}{ 嵒 } & & 6 & 7 & 8 & 9 & 10 & 11 & 12 & 13 & 14 & 15 & 16 & 17 & 18 & 19 & 20 & 21 & 22 & 23 & 24 \\
\hline & $\mathrm{N}$ & 15 & 14 & - & - & - & - & - & - & - & - & - & - & - & - & - & - & - & - & - \\
\hline & $\bar{x}$ & 126,3 & 143,2 & 162,5 & 180,5 & 205,0 & 125,7 & 248,7 & 267,0 & 290,5 & 312,5 & 336,6 & 362,0 & 381,2 & 404,4 & 428,3 & 457,5 & 473,0 & 479,0 & 490,0 \\
\hline & \pm & 12,2 & 19,0 & 14,7 & 14,7 & 16,6 & 19,2 & 18,1 & 16,4 & 15,6 & 15,8 & 16,8 & 18,4 & 18,1 & 18,8 & 16.4 & 21,4 & 24,4 & 26,1 & 24,2 \\
\hline \multirow{3}{*}{ 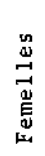 } & $\mathrm{N}$ & 28 & - & - & - & - & - & - & - & - & - & - & - & - & - & - & - & - & - & $\sim$ \\
\hline & $\bar{x}$ & 118,8 & 133,2 & 154,3 & 165,4 & 181,5 & 200,4 & 217,2 & 233,1 & 249,8 & 268,2 & 286,4 & 305,9 & 318,1 & 333,1 & 345,9 & 364,4 & 373,9 & 383,6 & 387,8 \\
\hline & \pm & 8,4 & 10,3 & 6,2 & 8,6 & 10,0 & 10,0 & 9,4 & 10,5 & 11,1 & 11,3 & 11,3 & 11,5 & 12,7 & 14,0 & 16,0 & 16,0 & 16,8 & 16,6 & 15,6 \\
\hline
\end{tabular}

TABLEAU $\mathrm{N}^{\circ} \mathrm{V}$

Gains de poids moyen par mois et journaliers des animaux extériorisës

\begin{tabular}{|c|c|c|c|c|c|c|c|c|c|c|c|c|c|c|c|c|c|c|c|}
\hline \multirow{3}{*}{ 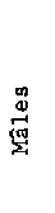 } & \multirow[b]{2}{*}{$\begin{array}{l}\text { Par } \\
\text { mois }\end{array}$} & $6-7$ & $7-8$ & $8-9$ & $9-10$ & $10-11$ & $11-12$ & $12-13$ & $13-14$ & $14-15$ & $15-16$ & $16-17$ & $17-18$ & $18-19$ & $19-20$ & $20-21$ & $21-22$ & $22-23$ & $23-24$ \\
\hline & & 16,9 & 19,3 & 18,0 & 24,5 & 20,7 & 23,0 & 18,0 & 23,5 & 22,0 & 24,1 & 25,4 & 19,2 & 23,2 & 23,9 & 29,2 & 15,5 & 6,0 & 11,0 \\
\hline & $\begin{array}{l}\text { Par } \\
\text { jour }\end{array}$ & 563,3 & 643,3 & 600,0 & 816,6 & 690,0 & 766,6 & 610,0 & 783,3 & 733,3 & 803,3 & 846,6 & 640,0 & 773,3 & 796,6 & 973,3 & 516,6 & 200,0 & 370,0 \\
\hline \multirow{2}{*}{ 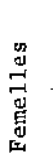 } & $\begin{array}{l}\text { Par } \\
\text { mois }\end{array}$ & 14,4 & 21,1 & 11,1 & 15,9 & 19,1 & 16,8 & 15,9 & 16,7 & 18,4 & 18,2 & 19,5 & 12,2 & 15,0 & 12,8 & 18,5 & 9,5 & 9,7 & 4,2 \\
\hline & $\begin{array}{l}\text { Par } \\
\text { jour }\end{array}$ & 480,0 & 703,3 & 370,0 & 530,0 & 636,6 & 560,0 & 530,0 & 556,6 & 613,3 & 606,6 & 650,0 & 406,6 & 500,0 & 426,6 & 616,6 & 316,6 & 323,3 & 140,0 \\
\hline
\end{tabular}


Comme chez les mâles, la diminution de la vitesse de croissance se retrouve vers le $21^{\mathrm{e}}$ mois.

\section{CONCLUSION}

La vitesse journalière de croissance chez les animaux Gobra garde jusqu'à 24 mois une valeur extrêmement intéressante tant chez les mâles que chez les femelles. Cependant il faut noter que le taux de croissance diminue de façon assez importante à partir du $21^{\mathrm{e}}$ mois. $\mathrm{Si}$ classiquement le poids au sevrage représente 40 p. 100 de celui de l'âge adulte dans cette expérience, il est de 25 p. 100 à 24 mois. Ces animaux pourront encore prendre du poids, mais il semble que, dans le cadre d'une alimentation intensive et à volonté dès Ia naissance, les capacités de croissance rapide soient épuisées vers le $21^{\mathrm{e}}$ mois. Par conséquent l'âge d'abattage se situerait un peu avant deux ans.

\section{Troisième partie}

\section{RESULTATS DES PREMIERS ABATTAGES}

A partir du $6^{\circ}$ mois, les animaux continuent à disposer à volonté du foin et de la paille de la station, ainsi que du concentré auquel ils ont été habitués depuis leur plus jeune âge - titrant, rappelons le, 0,9 UF et de 115 à $120 \mathrm{~g}$ de matières azotées par kilogramme.

Ce sont les résultats constatés sur les carcasses des premiers animaux abattus alors qu'ils avaient entre 27 à 30 mois, qui font l'objet de ce qui suit :

Trois taurillons ont été choisis en fonction de leur gain de poids entre leur naissance et deux ans, celui présentant le gain maximal, celui s'étant le moins développé et le troisième représentant le gain moyen constaté pour l'ensemble des animaux d'expérience.

TABLLAL $N^{\circ} \mathrm{VI}$

Donnëes gênérales concernant les trois anımaux

\begin{tabular}{|c|c|c|c|c|c|}
\hline $\mathrm{N}^{\circ}$ & $\begin{array}{c}\text { Date } \\
\text { naissance }\end{array}$ & $\begin{array}{c}\text { Poids } \\
\text { naissance } \\
(\mathrm{kg})\end{array}$ & $\begin{array}{c}\text { Poids à } \\
24 \mathrm{mois}\end{array}$ & $\begin{array}{c}\text { Poids vif } \\
\text { et (âge) à } \\
\text { I abattage }\end{array}$ & $\begin{array}{c}\text { Gann de } \\
\text { poids total } \\
\text { (kg) }\end{array}$ \\
\hline 2717 & 24.5 .68 & 20 & 560 & $\begin{array}{c}589 \mathrm{~kg} \\
(30 \mathrm{mois})\end{array}$ & 569 \\
\hline 2734 & 24.6 .68 & 17 & 481 & $\begin{array}{c}556 \mathrm{~kg} \\
(29 \mathrm{mois})\end{array}$ & 539 \\
\hline 2748 & 21.8 .68 & 26 & 418 & $440 \mathrm{~kg}$ & 620 \\
\hline
\end{tabular}

\section{OBSERVATIONS}

Les observations et calculs suivants sont effectués (4) :

- Poids vif avant le jeûne, à l'arrivée à Dakar.

- Poids vif après $24 \mathrm{~h}$. de jeûne au moment de l'abattage.

- Poids de la carcasse chaude.

- Poids du contenu de la panse.

- Poids du $5^{\mathrm{e}}$ quartier (tête, cuir, viscères, pieds).

- Poids des demi-carcasses après $24 \mathrm{~h}$. de ressuyage en chambre froide (queue et rognon compris).

- Longueur de la carcasse (bord antérieur de la symphyse pubienne - milieu bord antérieur de la première côte).

- Epaisseur de la cuisse (au sommet d'un triangle isocèle dont la symphyse pubienne est la base).

- Epaisseur des muscles du plat de côtes (au niveau du $7^{\mathrm{e}}$ espace intercostal sur la ligne matérialisée par la mesure de longueur).

N.B. : Ces trois mesures sont prises après 24 heures de ressuyage.

- Jugement à l'œil :

- conformation de la carcasse;

- état du gras de carcasse;

- état de la graisse de couverture;

- état de la graisse de rognon;

- état de la graisse musculaire. 
- Calcul du rendement et du rendement vrai :

- rendement $=\frac{\text { Poids carcasse chaude }}{\text { Poids vif (après } 24 \mathrm{~h} \text { de jeûne) }} \times 100$

- rendement vrai $=\frac{\text { Poids carcasse chaude }}{\text { Poids vif (après } 24 \mathrm{~h} \text { de jeûne) - poids contenu de panse }}$

- La demi-carcasse gauche ressuyée est découpée de la façon suivante:

- demi-bosse;

- gras de rognon;

- épaule : collier - paleron (une partie du collier enlevée avec la tête au niveau de la $3^{2}$ vertèbre cervicale constitue la part coutumière qui comprend en outre la hampe et l'onglet);

- pis de bouf comprenant:

- le gros bout de poitrine allant jusqu'à la $3^{\mathrm{e}}$ côte,

- la poitrine : 4" à la $7^{*}$ côte,

- le tendon et le flanchet;

- panneau;

- le pan découpé en :

- train de côtes, séparé au $10^{\circ}$ espace intercostal,

- aloyau et cuisse;

- indice de gras $=$

$\frac{\text { Poids gras de rognon }}{\text { Poids carcasse froide }} \times 100$

TABLEAU $N^{\circ} \mathrm{VII}$

Rësultats des abattages

Poids avant jê̂ne

Poids après jeûne

Pourcentage perte au jeûne

Poids carcasse chaude

Pouds carcasse froide

Pourcentage perce au ressuyage

Rendement

Rendement vrai

Pourcentage contenu panse

Pourcentage 5ème quartier

En pourcentage du poids de la carcasse frolde

- êpaule

- pis

- panneau

- train de côtes

- globe

- bosse

- gras de rognon

Longueur carcasse

Eparsseur cuisse

Eparsscur plat de côtes

Indice de gras

Indlce de compacitê

Conformation de la carcasse

Gras de carcasse

Graisse de couverture

Gras de rognon

Craisse musculaze

\begin{tabular}{|c|c|c|}
\hline 2717 & 2734 & 2748 \\
\hline 589 & 556 & 440 \\
\hline 580 & 542 & 430 \\
\hline 1,52 & 2,51 & 2,27 \\
\hline 355 & 326 & 264 \\
\hline 351 & 323 & 263 \\
\hline 1,12 & 0,92 & 0,37 \\
\hline 64,72 & 62,88 & 63,72 \\
\hline 67,48 & 66,06 & 65,58 \\
\hline 4,08 & 4,81 & 2,83 \\
\hline 24,89 & 25,53 & 27,32 \\
\hline 21,30 & 23,20 & 18,93 \\
\hline 12,15 & 9,81 & 21,51 \\
\hline 5,68 & 6,48 & 7,19 \\
\hline 10,51 & 10,55 & 10,0 \\
\hline 44,03 & 43,33 & 44,39 \\
\hline 3,40 & 3,33 & 2,50 \\
\hline 1,93 & 2,28 & 4,24 \\
\hline 128,0 & 125,5 & 116,5 \\
\hline 29,6 & 29,7 & 27,4 \\
\hline 4,50 & 5,0 & 4,75 \\
\hline 1,94 & 2,29 & 4,26 \\
\hline 2,74 & 2,57 & 2,26 \\
\hline 5 & 4,5 & 4 \\
\hline 4 & 4 & 4 \\
\hline 4,5 & 4 & 5 \\
\hline 5 & 4,5 & 5 \\
\hline 3 & 3 & 3 \\
\hline
\end{tabular}


- indice de compacité $=$

Poids carcasse

Longueur carcasse

- Pour le jugement à l'œil des carcasses, les cotations suivantes sont adoptées:

0 - nul

1 - médiocre à très faible

2 - faible

3 - moyen

4 - bon, satisfaisant

5 - très bon.

\section{RESULTATS ET DISCUSSIONS}

Ils apparaissent aux tableaux $\mathrm{n}^{\text {ss }} 6$ et 7 .

Les gains de poids sont remarquables et montrent bien que les qualités bouchères de cette race déjà signalées (3) sont intéressantes.

La demi-carcasse droite de l'animal $n^{\circ} 2748$ est découpée selon la méthode pratiquée à Dakar :

- quartier avant, comprenant le paleron, le restant du collier et une partie du pis.

- quartier arrière, comprenant le reste de la demi-carcasse.

Le quartier arrière est traité par un boucher de la place de Dakar le 19 novembre 1970. Cet arrière pèse $95,3 \mathrm{~kg}$.

Les résultats apparaissent au tableau $\mathrm{n}^{\circ} 8$.

TABLEAU $N^{\circ} \mathrm{VIII}$

\begin{tabular}{|c|c|c|}
\hline \multirow[b]{2}{*}{ Détail des morceaux } & \multicolumn{2}{|c|}{ Poids en kg } \\
\hline & Détail & Par groupe \\
\hline $\begin{array}{l}1 \text { - Cuisse } \\
\text { Tente de tranche } \\
\text { Gite } \\
\text { Jarret } \\
\text { Tranche grasse }\end{array}$ & $\begin{array}{l}7,5 \\
9,7 \\
2,7 \\
4,9\end{array}$ & 24,8 \\
\hline 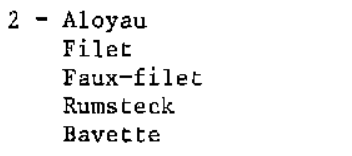 & $\begin{array}{l}2,0 \\
9,3 \\
4,7 \\
1,5\end{array}$ & 17,5 \\
\hline 3 - Plat de côtes & 16,5 & \\
\hline $\begin{array}{l}4 \text { - Gros bout de } \\
\text { poitrine }\end{array}$ & 13,0 & \\
\hline $5-0 s$ & 9,5 & \\
\hline 6 - Graisse + déchets & 10,0 & \\
\hline 7 - Gras rognon + rognon & 4,0 & \\
\hline
\end{tabular}

Il est intéressant de comparer ces différents résultats à ceux obtenus lors d'essais d'engraissement intensif de zébus Peulhs sénégalais. La comparaison peut se faire soit avec des animaux d'âges les plus proches possibles (taurillons de 3 à 5 ans avant et après embouche), soit avec des animaux dont le poids vif et surtout la longueur des carcasses se rapproche de ceux des animaux " extériorisés ". Ces données figurant au tableau $n^{\circ} 9$ sont extraites des études de CALVET et collab. (1) et VALENZA et collab. (4) et peuvent servir de " témoins ".

A la lecture de ces tableaux, on constate que :

1. Le pourcentage de perte au jeûne, assez faible, n'a pas grande signification dans les conditions de l'expérience, puisque les animaux sont à jeun depuis leur départ de Dara.

2. Le pourcentage de perte au ressuyage est faible par rapport aux "témoins " $(1,3$ à 2,2$)$ et celui donné en Europe (1,5 à 4 p. 100 après 48 heures de ressuyage). Il faut noter que le ressuyage n'est que de 24 heures pour les animaux considérés.

Cette faible perte est la conséquence d'une importante graisse de couverture empêchant l'évaporation. Elle est la plus faible chez l'animal le plus gras (2.748).

D'autres facteurs agissent évidemment sur cette mesure: la température ambiante, le degré d'humidité, la vitesse de l'air et le temps écoulé depuis l'abattage. Mais dans tous les cas, la durée et les conditions de ressuyage sont identiques.

3. Les rendements sont excellents et dépassent très largement ceux des animaux tout venant $(50$ p. 100$)$ et ceux des animaux d'embouche intensive (55 p. 100) (2). Ces chiffres sont comparables à ceux obtenus en Europe sur veau gras: 65 p. 100 et jeunes bœufs gras : 60 p. 100.

4. Le pourcentage de contenu de panse est très faible. Ceci s'explique par la composition de la ration consommée par les animaux. Ils reçoivent du concentré et de l'eau à volonté et finalement consomment très peu d'aliment de lest sous forme de paille ou de foin de brousse. De plus, ces animaux sont jeunes et l'on sait que proportionnellement au poids total, le volume du contenu digestif est plus important chez les bovins adultes. 
TABLEAU $\mathrm{N}^{\circ} \mathrm{IX}$

Caractérıstiques des carcasses d'animaux d'embouche

\begin{tabular}{|c|c|c|c|c|}
\hline & $\begin{array}{l}\text { Taurillons } \\
3 / 5 \text { ans avant } \\
\text { embouche }\end{array}$ & $\begin{array}{c}\text { T'aurillons } \\
3 / 5 \text { ans aprẽs } \\
4 \text { mols d'embouche }\end{array}$ & $\begin{array}{l}\text { Boeufs } \\
7 / 9 \text { ans avant } \\
\text { embouche }\end{array}$ & $\begin{array}{c}\text { Boeufs } \\
7 / 9 \text { ans après } \\
4 \text { mols d'embouche }\end{array}$ \\
\hline Poids avant jeûne & 265 & 385 & - & 425,8 \\
\hline Poids apcès $24 \mathrm{~h}$, de jeûne & 254 & 358 & 314,4 & 406,2 \\
\hline Pourcentage perte au jeûne & 4,02 & 7,01 & - & 4.58 \\
\hline Poids carcasse chaude & 131,2 & 200,2 & 161,4 & 224 \\
\hline Poids carcasse froide & 128,7 & 197,1 & 157,9 & 221 \\
\hline Pourcentage perte au ressuyage & 1,91 & 1,56 & 2,19 & 1,34 \\
\hline Rendement & 51,65 & 55,92 & 51,44 & 55,18 \\
\hline Rendement vrai & 56,73 & 60,05 & 58,26 & 59,68 \\
\hline Pourcentage contenu panse & 12,58 & 6,89 & 11,76 & 7,89 \\
\hline $\begin{array}{l}\text { Pourcentage sème quartier } \\
\text { En pourcentage poids } \\
\text { carcasse froide }\end{array}$ & 27,71 & 27,85 & 30,48 & 26,77 \\
\hline - êpaule & 26,75 & 23,71 & 21,43 & 19,93 \\
\hline . pis & 13,18 & 13,51 & 14,67 & 15,43 \\
\hline - panneau & 7,51 & 5,38 & 6,18 & 4,65 \\
\hline - train de côtes & 7,24 & 9,34 & 10,42 & 10,55 \\
\hline - globe & 43,13 & 42,83 & 43,32 & 42,40 \\
\hline - bosse & 1,45 & 2,32 & 0,78 & 1,31 \\
\hline - gras rognon & 0,51 & 2,92 & 0,70 & 2,84 \\
\hline Longueur de la carcasse & 107,8 & 113 & $12 \bar{\jmath}, 5$ & 122,2 \\
\hline Epaisseur cuisse & 19,4 & 23 & 20,22 & 25,07 \\
\hline Epaisseur plat de côtes & 1,9 & 2,9 & 1,86 & 3,04 \\
\hline Indice de gras & 0,51 & 2,92 & 0,70 & 2,84 \\
\hline Indice de compacité & 1,19 & 1.77 & 1,26 & 1,81 \\
\hline
\end{tabular}

5. L'indice de gras est élevé en particulier pour la carcasse $n^{\circ} 2.748$ dont le gras de rognon est envahissant.

6. Les animaux bien que non castrés ont une viande assez claire. Il en est de même pour la graisse, contrairement à ce que l'on observe sur la majorité des carcasses de zébus provenant de l'élevage traditionnel.

Le persillé et le marbré sont également présents.

7. Qualités organoleptiques :

\section{Tendreté :}

Bien qu'il s'agisse de mâles et par conséquent d'animaux dont classiquement les fibres musculaires sont de diamètre important et la teneur en fibres élastiques relativement plus élevée que chez les femelles, la tendreté est dans l'ensemble jugée " très bonne ".
Ceci est dû à divers facteurs dont en particulier le jeune âge des animaux, le très bon état d'engraissement, le service (ces animaux n'effectuent depuis leur naissance que des déplacements peu importants).

\section{Caractéristiques gustatives:}

Il n'est pas fait d'étude systématique par un jury spécialisé ou même des jury familiaux, mais les qualités organoleptiques sont appréciées par dégustation d'entrecôtes provenant de l'animal $n^{\circ} 2.734$. Dans l'ensemble, la qualité de la viande est appréciée principalement à cause du persillé abondant, de sa jutosité, de sa tendreté et de son goût.

8. Dans l'ensemble, ces carcasses sont nettement supérieures à celles obtenues après une embouche de quatre mois. La cuisse est plus épaisse, le pourcentage du poids de globe plus ćlevé. On peut leur reprocher leur excès de graisse comme en témoignent les pourcentages 
des poids de la bosse et du gras de rognon; mais le marbré et le persillé sont plus abondants.

\section{CONCLUSIONS}

Les animaux "d'extériorisation " donnent à l'abattage d'excellentes carcasses avec cependant une quantité de gras un peu trop importante qui, selon les bouchers de la place, a tendance à " manger la viande " .

Les rendements observés, 63 p. 100 en moyenne, sont nettement plus élevés que ceux enregistrés sur les animaux provenant soit de l'élevage traditionnel, soit d'un parc d'embouche.

II faut noter que, malgré la possibilité donnée à ces animaux de développer au maximum leurs masses musculaires, les défauts classiques de la carcasse de zébu subsistent, en particulier une faible convexité de la cuisse, un développement insuffisant de la croupe et du dessus, c'est-à-dire en fait une certaine faiblesse des arrières.

Au cours de cette expérience, les notions économiques n'entrent pas en ligne de compte puisqu'il s'agit littéralement de faire " éclater " les animaux.

Ces résultats permettent d'autre part de déterminer le sens des prochains travaux à effectuer sur ces animaux : amélioration de la conformation bouchère en insistant sur la qualité des arrières, recherche de rations permettant d'éviter le travers " excès de gras ", recherche de l'âge optimal d'abattage.

\title{
SUMMARY
}

\section{Phenotypic potentialities of zebu Gobra}

\begin{abstract}
Three bulls of an herd of which phenotypic potentialities have been expressed by a rationnal feeding from birth are slaughtered at 30,29 and 27 months old. Their respective live weights are 589,556 and $440 \mathrm{~kg}$. Their daily growth is 632,620 and $510 \mathrm{~g}$. The outputs are $64,7,62,8$ and 63,7 p. 100 . The carcasses are better than these coming from the usual cattle or feed-lots.
\end{abstract}

\section{RESUMEN}

Exteriorización de los potenciales genéticos del cebú Peulh de Senegal (Gobra)

Se mataron a 30,29 y 27 meses de edad tres toritos perteneciendo a un lote de animales cuyos potenciales genéticos se exteriorizaron por una alimentación racional desde su nacimiento. Son de 589,556 y $440 \mathrm{~kg}$ sus pesos respectivos representante un aumento medio diario de 632,620 y $510 \mathrm{~g}$. Son de $64,7,62.8$ y 63,7 los rendimientos. Las canales obtenidas son superiores a las de animales proviniendo de la ganaderia tradicional o de majada de engorde.

\section{BIBLIOGRAPHIE}

1. DENIS (J. P.) et VALENZA (J.), « Etude et sélection du zébu peulh sénégalais », Communication à la $2 \mathrm{e}$ conférence mondiale de production animale, Maryland (U.S.A.), 14-20 juillet 1968.

2. DENIS (J. P.) et VALENZA (J.), «Le comportement pondéral des femelles adultes de race Gobra (zébu peulh sénégalais). Comparaison avec les animaux importés pakistanais et Guzera », Rev. Elev. Méd. vét. Pays trop. 1970, 23 (2): 229-41.

3. DENIS (J.P.) et VALENZA (J.), \& Influence du niveau alimentaire sur la croissance du zébu peulh sénégalais (Gobra) », Communication au congrès mondial vétérinaire. Mexico, août 1971.
4. DENIS (J. P.) et VALENZA (J.), « Extériorisation des potentialités du zébu Gobra. I. De la naissance au sevrage. Communication au Colloque OCAM sur l'Elevage. Fort-Lamy 8-13 déc. 1969.

5. « Rapports annuels du Centre de Recherches zootechniques de Dahra-Djoloff (Sénégal)».

6. REDON (A.), \& Note sur la valeur zootechnique du zébu sénégalais ", Rev. Elev. Méd. vét. Pays trop., 1962, 15 (3) : 265-71.

7. VALENZA (J.), CALVET (H.) et ORUE (J.), "Essais d'engraissement du zébu peulh sénégalais (Gobra) », Dakar-Hann, I.E.M.V.T., Laboratoire national de l'Elevage, février 1970, 36 p. 